\title{
T1 mapping is abnormal before decline in EF in patients with Becker and Duchenne muscular dystrophy
}

\author{
Jonathan H Soslow ${ }^{1 *}$, Bruce M Damon ${ }^{3}$, William B Burnette², David Parra', Larry W Markham ${ }^{4}$ \\ From 16th Annual SCMR Scientific Sessions \\ San Francisco, CA, USA. 31 January - 3 February 2013
}

\section{Background}

Patients with Duchenne muscular dystrophy (DMD) develop cardiomyopathy (CM) at an earlier age compared to Becker muscular dystrophy (BMD), but the age of onset within each diagnosis is variable. A method to predict $\mathrm{CM}$ onset associated with dystrophinopathy could alter therapeutic approaches and improve outcomes. Shortened post-contrast T1 relaxation times are an early marker of myocardial fibrosis and are abnormal in patients with DMD as described in our previous work. To our knowledge, T1 mapping has not been evaluated in BMD patients. We hypothesized that patients with BMD would also have abnormal T1 times and that these abnormalities would precede LV dysfunction.

\section{Methods}

Twenty-six CMR scans from these 2 dystrophinopathy populations ( $\mathrm{N}=5 \mathrm{BMD}$ and $\mathrm{N}=21 \mathrm{DMD})$ were compared with 10 CMR scans from control patients without cardiovascular disease. T1 maps were created from the Look-Locker sequence, obtained 10 minutes after gadolinium injection, using MRMap. Using MatLab, T1 times were obtained for every voxel in 6 standard myocardial segments in the short axis at the level of the papillary muscles. Mean T1 times were compared between BMD, DMD and controls using a Kruskal Wallis test. A subset of patients with normal LVEF and dystrophinopathy (BMD or DMD) was compared to controls using a Mann-Whitney U test.

\section{Results}

The mean age of patients was $25.4 \pm 8.1$ years in the BMD group, $15.7 \pm 4.3$ years in the DMD group, and $16.9 \pm 1.3$ years in controls. One patient with BMD and 13 patients with DMD had LVEF $<55 \%$. Two patients from both BMD and DMD had LV dilatation. BMD and DMD patients had significantly shorter mean postcontrast T1 compared with controls (BMD: $355 \mathrm{~ms}$, 95\% CI $(314,395)$, DMD:357 ms, 95\% CI $(323,390)$, control: $420 \mathrm{~ms}, 95 \% \mathrm{CI}(389,451), \mathrm{p}=0.035)$ (Figure 1). The subset of BMD and DMD patients and normal LVEF also had a significant decrease in post-contrast $\mathrm{T} 1$ when compared to controls $(-52.4 \mathrm{~ms}, 95 \%$ CI $(-7.2$, -97.5), $\mathrm{p}=0.018$ ) (Figure 2).

\section{Conclusions}

Myocardial post-contrast $\mathrm{T} 1$ relaxation times in BMD and DMD are significantly shorter than in controls. They remain significantly decreased in the subset of patients with normal LVEF, suggesting that CMR may have prognostic utility in detecting subclinical myocardial damage associated with loss of dystrophin prior to a decline in LVEF. Further longitudinal study is necessary to evaluate whether $\mathrm{T} 1$ times can predict future onset of $\mathrm{CM}$ in BMD and DMD.

\section{Funding}

none. 


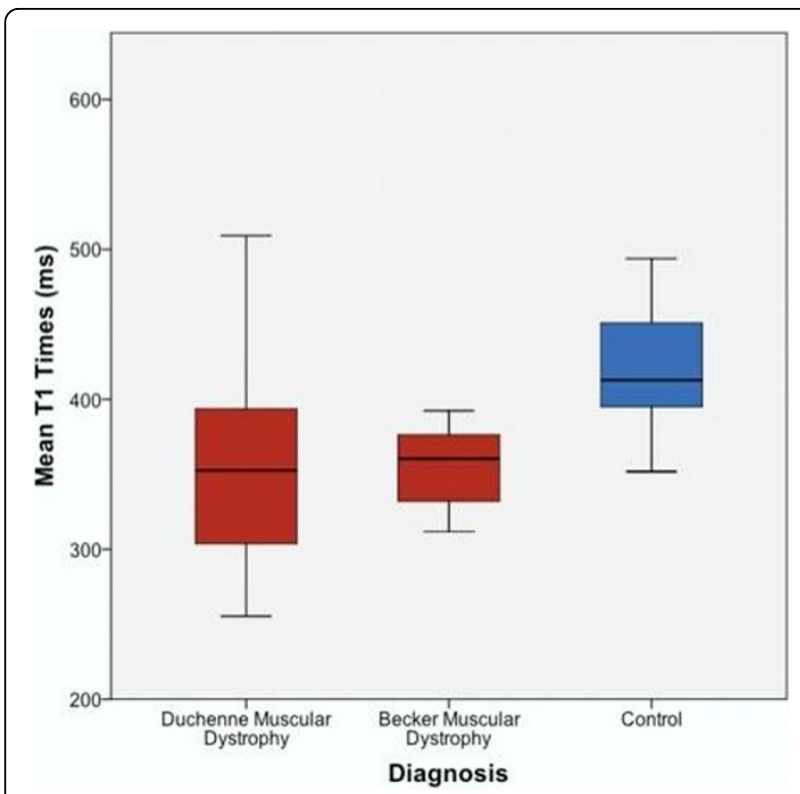

Figure 1 Mean T1 times by diagnosis. Patients with Duchenne muscular dystrophy (DMD) and Becker muscular dystrophy (BMD) have significantly shorter mean post-contrast $\mathrm{T} 1$ relaxation times compared with controls.

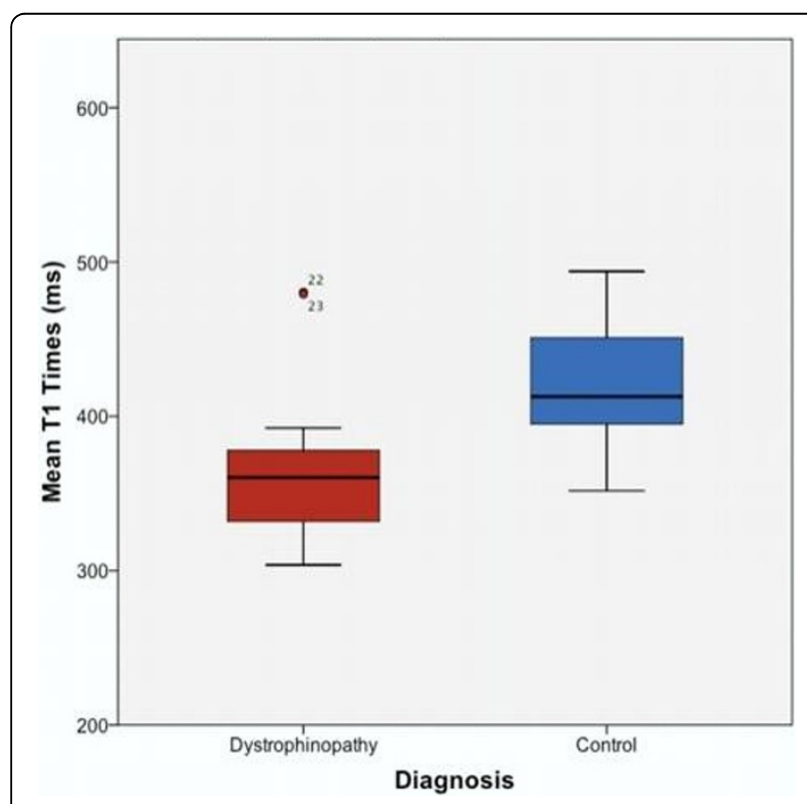

Figure 2 Mean $T 1$ times in patients with normal LVEF and dystrophinopathy compared to controls. A subset of patients with Becker muscular dystrophy (BMD) and Duchenne muscular dystrophy (DMD) and normal left ventricular ejection fraction (LVEF) also had a significant decrease in post-contrast $\mathrm{T} 1$ when compared to controls. This suggests that myocardial T1 mapping can detect myocardial damage in patients with dystrophinopathy prior to a decline in LVEF.

\section{Author details}

${ }^{1}$ Pediatrics, Division of Pediatric Cardiology, Vanderbilt University Medical Center, Nashville, TN, USA. ${ }^{2}$ Neurology, Vanderbilt University Medical Center, Nashville, TN, USA. ${ }^{3}$ Radiolology and Radiological Sciences, Molecular

Physiology and Biophysics, and Biomedical Engineering, Vanderbilt University Medical Center, Nashville, TN, USA. ${ }^{4}$ Internal Medicine and Pediatrics, Division of Cardiology, Vanderbilt University Medical Center, Nashville, TN, USA.

Published: 30 January 2013

doi:10.1186/1532-429X-15-S1-P149

Cite this article as: Soslow et al: T1 mapping is abnormal before decline in EF in patients with Becker and Duchenne muscular dystrophy. Journal of Cardiovascular Magnetic Resonance 2013 15(Suppl 1): P149.

\section{Submit your next manuscript to BioMed Central and take full advantage of:}

- Convenient online submission

- Thorough peer review

- No space constraints or color figure charges

- Immediate publication on acceptance

- Inclusion in PubMed, CAS, Scopus and Google Scholar

- Research which is freely available for redistribution 